\title{
Contents
}

$1 \quad$ Introduction ...........................................

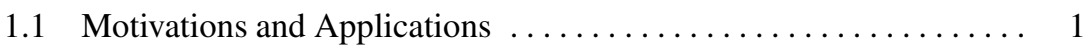

1.2 Mathematical Framework ............................

1.3 Book Chapters ...................................

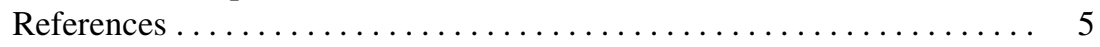

\section{Part I: Mathematical Theory}

2 Mathematical Preliminaries ............................ 11

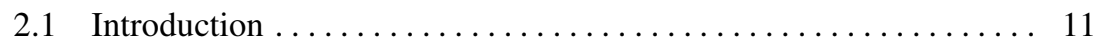

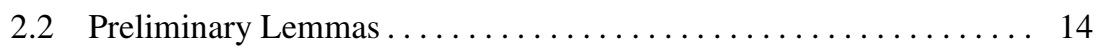

2.3 Implicit Function Theorems ....................... 14

2.4 Linear Algebra ......................................

2.5 Functions with Bounded Variation $\ldots \ldots \ldots \ldots \ldots \ldots \ldots \ldots \ldots \ldots$

References .......................................

3 One-Dimensional Scalar Conservation Laws ................ 23

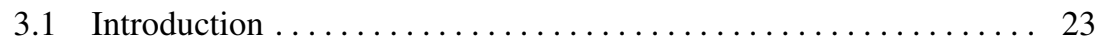

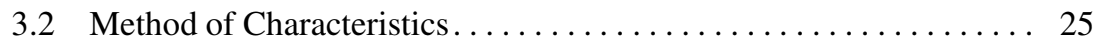

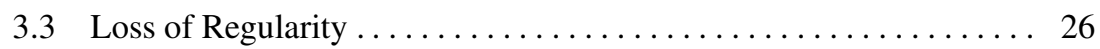

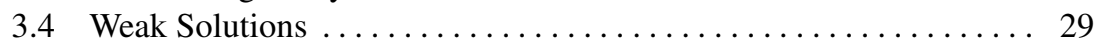

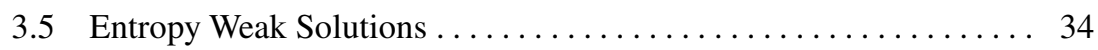

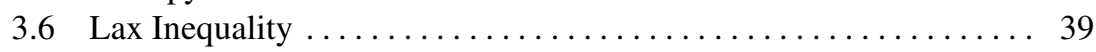

References ....................................... 42

4 The Riemann Problem ................................ 43

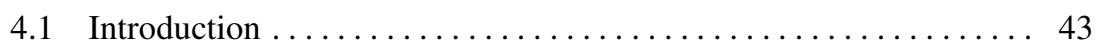

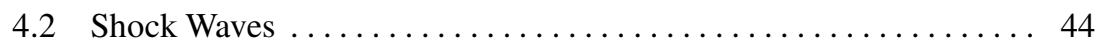

4.3 Non-entropy Shock Waves ......................... 45

4.4 Rarefaction Waves .................................. 45

4.5 Contact Waves ..................................... 


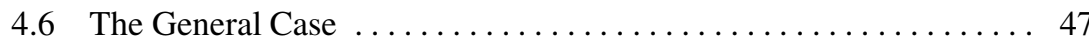

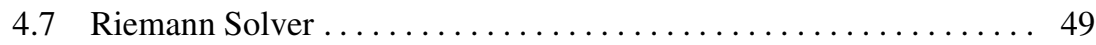

References .............................. 50

5 The Cauchy Problem $\ldots \ldots \ldots \ldots \ldots \ldots \ldots \ldots \ldots \ldots \ldots \ldots \ldots \ldots$

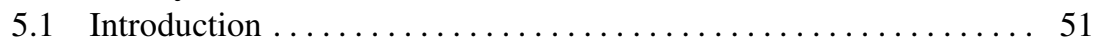

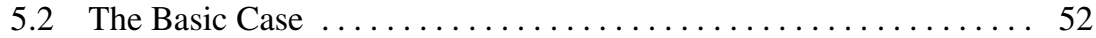

5.3 The General Case ........................... 54

5.3.1 Approximation of the Initial Data .............. 54

5.3 .2 Approximation of the Flux ................. 55

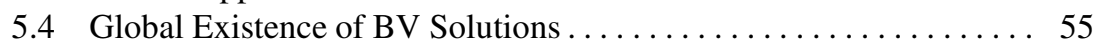

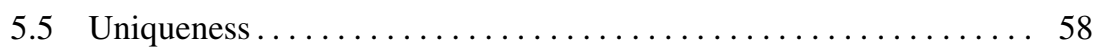

References ................................. 62

6 The Initial-Boundary Value Problem and the Constraint ......... 63

6.1 Introduction $\ldots \ldots \ldots \ldots \ldots \ldots \ldots \ldots \ldots \ldots \ldots \ldots \ldots \ldots \ldots$

6.2 The Initial-Boundary Value Problem . . . . . . . . . . . . . . . . 64

6.3 The Constrained Riemann Problem . . . . . . . . . . . . . . . . . . . . . . 74

6.4 The Constrained Cauchy Problem ... . . . . . . . . . . . . . . 79

6.5 The Constrained Initial-Boundary Value Problem ........... 87

References .............................. 91

7 One-Dimensional Systems of Conservation Laws ............. 93

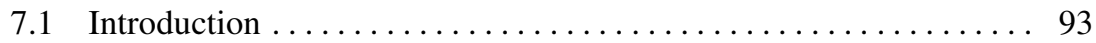

7.2 Strictly Hyperbolic Linear Systems with Constant Coefficients .... 98

7.3 Riemann Problems ... . . . . . . . . . . . . . . . . . . . . . . 100

7.3.1 Rarefaction Waves .............................. 100

7.3.2 Shock Waves and Contact Discontinuities .............. 102

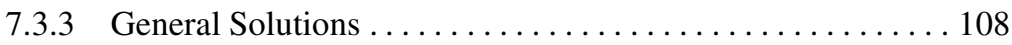

References ................................. 110

8 One-Dimensional Systems of Balance Laws (Weakly Coupled) ..... 111

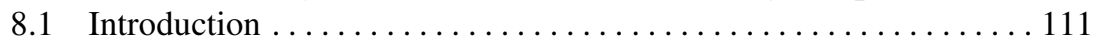

8.2 The Convective Part ... . . . . . . . . . . . . . . . . . . . . 113

8.3 The Non-local Source Term ....................... 115

8.4 Operator Splitting . . . . . . . . . . . . . . . . . . . . 117

8.5 Well Posedness of the Cauchy Problem .................. 118

References .................................... 119

\section{Part II: Models for Vehicular Traffic}

$9 \quad$ Vehicular Traffic $\ldots \ldots \ldots \ldots \ldots \ldots \ldots \ldots \ldots \ldots \ldots \ldots \ldots \ldots$

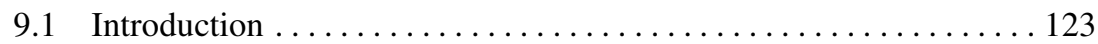

9.2 Mathematical Models ... . . . . . . . . . . . . . . . . . . . . . 124

9.3 Computational Models . . . . . . . . . . . . . . . . . . . . . 127 
9.4 The Fundamental Macroscopic Traffic Variables . . . . . . . . . . . . . 130

9.5 Relations between the Fundamental Traffic Variables . . . . . . . . . . 132

References ...................................... 134

10 Equilibrium Traffic Models $\ldots \ldots \ldots \ldots \ldots \ldots \ldots \ldots \ldots \ldots \ldots \ldots$

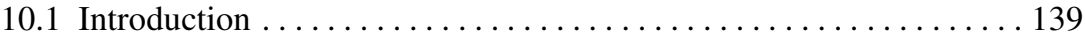

10.2 Riemann Problems . . . . . . . . . . . . . . . . . . . . . . . . 143

10.3 The Drawbacks of the Equilibrium Traffic Models . . . . . . . . . . 147

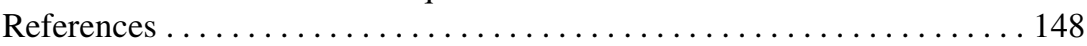

11 Generalizations of Equilibrium Traffic Models ................ 149

11.1 Introduction . . . . . . . . . . . . . . . . . . . . . . . . 149

11.2 Highway with an Entrance and Constraints .............. 149

11.3 Merging Roads .............................. 150

11.4 Traffic Circle . . . . . . . . . . . . . . . . . . . . . . . . . . 152

11.5 Multi-population ................................ 154

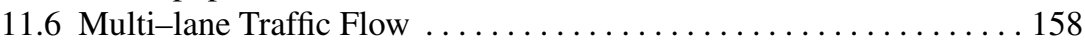

References ..........................................

12 Cost Functionals $\ldots \ldots \ldots \ldots \ldots \ldots \ldots \ldots \ldots \ldots \ldots \ldots \ldots \ldots \ldots \ldots$

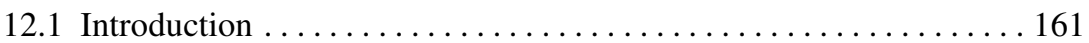

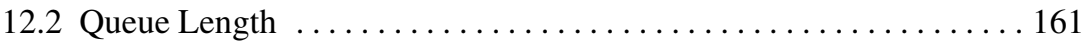

12.3 Stop and Go Waves . . . . . . . . . . . . . . . . . . . .

12.4 Travel Times ................................... 164

12.5 Density Dependent Functionals . . . . . . . . . . . . . . . 166

References .................................. 166

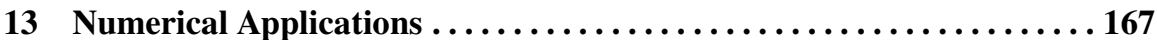

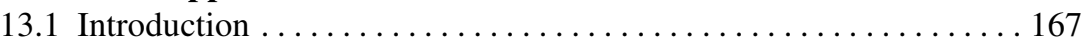

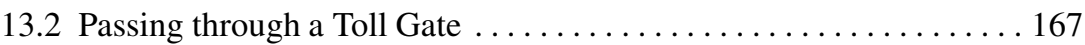

13.3 Lax-Friedrichs vs. Wave Front Tracking . . . . . . . . . . . . . . 169

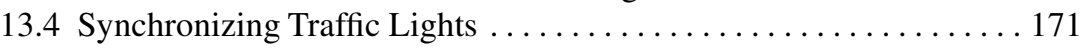

References ................................. 172

14 Non-equilibrium Traffic Models $\ldots \ldots \ldots \ldots \ldots \ldots \ldots \ldots \ldots \ldots \ldots$

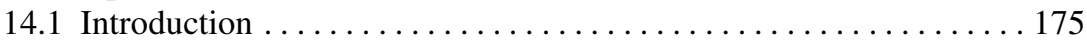

14.2 Generalized PW Models . . . . . . . . . . . . . . . . . . . . 175

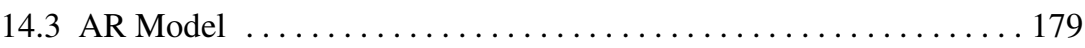

References ..................................... 190

\section{Part III: Models for Pedestrian Traffic}

15 General Concepts $\ldots \ldots \ldots \ldots \ldots \ldots \ldots \ldots \ldots \ldots \ldots \ldots \ldots \ldots \ldots \ldots \ldots$

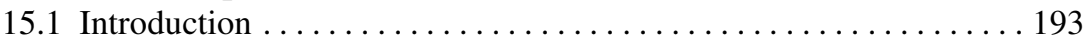

15.2 The Need of a Non-classical Theory . . . . . . . . . . . . . 197

References ...................................... 


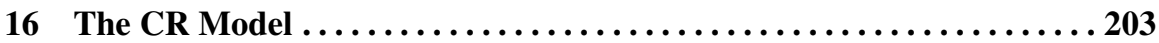

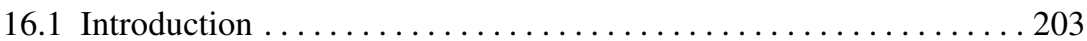

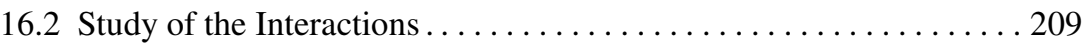

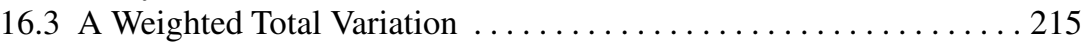

16.4 Numerical Example. . . . . . . . . . . . . . . . . . . . . . . . 218

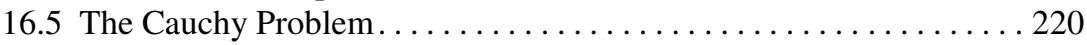

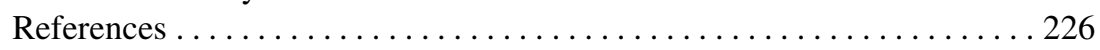

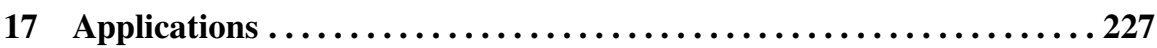

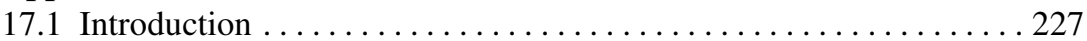

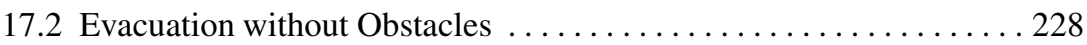

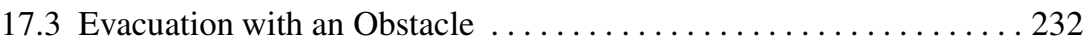

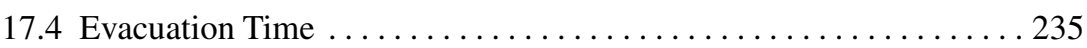

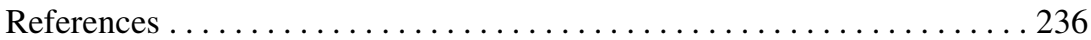

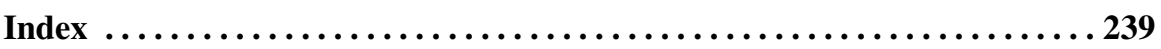

